Naturheilverfahren in der Schwangerschaft

\title{
Welche Phytotherapeutika und welche auf keinen Fall?
}

\author{
Von Schwangeren wird oft der Wunsch nach dem Einsatz von Natur- \\ heilverfahren an den Arzt herangetragen. Was dabei zu beachten ist, \\ erläutert Prof. Dr. A.-M. Beer in diesem Beitrag.
}

\section{$?$ Wann werden Phytotherapeutika \\ in der Schwangerschaft eingesetzt?}

Antwort: $\mathrm{Zu}$ den wesentlichen Indikationen zählen Obstipation, Emesis und Hyperemesis, depressive Verstimmungen und nervöse Unruhezustände.

Aufgrund fehlender Studien wurde die Anwendung einiger Phytotherapeutika bei Schwangerschaftserbrechen von der Kommission E ausgeschlossen. Ein Beispiel ist der Ingwerwurzelstock. In der traditionellen chinesischen Medizin ist er nicht kontraindiziert. Nach europäischen Maßstäben gilt er aber als ungenügend untersucht. Bei den chemisch-definierten Therapeutika ist die Datenlage vergleichbar schlecht. Aus der Erfahrung heraus wird Ingwer daher zur Antiemese in der Praxis eingesetzt. So ist eine kleine Menge frisch geriebener Ingwerwurzel in Karottensuppe zu empfehlen. Daneben stehen auch Teerezepturen zur Verfügung (Tab. 1).

\section{?elche Phytopharmaka sind bei Schwangeren explizit kontraindiziert?}

Antwort: Hierzu gehören Beerentraubenblätter, Chinarinde, Huflattichblätter,

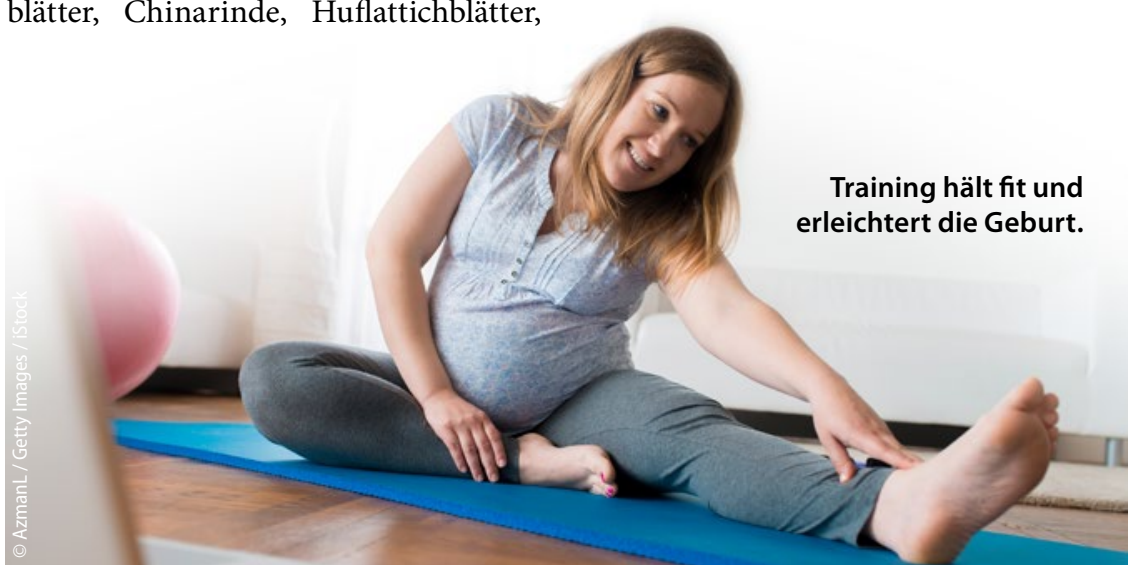

Tab. 1 Teerezeptur gegen Schwangerschaftserbrechen

- Matricariae flos. tot. (Kamillenblüten) $20,0 \mathrm{~g}$

- Melissae folium conc. (Melissenblätter) 20,0 g

- Menthae piperitae folium conc. (Pfefferminzblätter) $20,0 \mathrm{~g}$

- M. f. spec. antiemeticae

- D.S. 1 gehäuften TL Teemischung mit $150 \mathrm{ml}$ kochendem Wasser übergießen, ca. 10-15 Minuten ziehen lassen, abseihen. Bis zu 5 Tassen frisch bereiteten Tee tgl.

Kava-Kavawurzelstock, Rohpapain, Rauwolfiawurzel und Sonnenhutkraut. Sie alle sind wegen toxikologischer, embryotoxikologischer Wirkungen in der Schwangerschaft kontraindiziert. Einige Drogen erhöhen die kontraktile Aktivität der Uterusmuskulatur wie z.B. Keuschlammfrüchte, Laxanzien (Faulbaumrinde, Sennesblätter etc.), Petersilienwurzel, Wacholderbeeren bzw. ätherisches Wacholderbeeröl. Auch Süßholzwurzel ist kontraindiziert, sie kann zu hormonellen Veränderungen (Aldosteron, Renin) führen. 